\title{
A Comparative Study on the Effect of Low-Dose Oral Ketamine and Subcutaneous Ketamine on Post General Anesthesia Delirium and Pain in Children Undergoing Inguinal Hernia Surgery
}

\author{
Alireza Takzare ${ }^{1}$, Anahid Maleki ${ }^{1}$, Mehrdad Goudarzi $^{1}$, Alireza Ebrahim Soltani ${ }^{1} \&$ Irandokht Ghorbani Sepehr $^{1}$ \\ ${ }^{1}$ Anaesthesiology Department, Children's Hospital Medical Centre, Tehran University of Medical Sciences, \\ Tehran, Iran \\ Correspondence: Anahid Maleki, Anaesthesiology Department, Children's Hospital Medical Centre, Tehran \\ University of Medical Sciences, Tehran, Iran. E-mail: A-maleki@tums.ac.ir
}

Received: December 23, 2018

Accepted: January 6, 2019

Online Published: January 20, 2019

doi:10.5539/jmbr.v9n1p1

URL: https://doi.org/10.5539/jmbr.v9n1p1

\begin{abstract}
Objective: Acute pain is a common complication in children undergoing surgery. Acute pain and delirium are associated with agitated behavior, in which there is a risk that the patient causes harm to himself and others. Acute pain and delirium also dissatisfy parents and caregivers during recovery, prolong the recovery and hospitalization, and make more nursing care necessary.

Material and Methods: In this study, 90 ASA I children, aged 3-8, and two candidates for elective inguinal hernia surgery were divided into three groups based on a random number table using block size of four. Parental permission was obtained beforehand. In group $1,1 \mathrm{mg} / \mathrm{kg}$ of ketamine $(3 \mathrm{cc})$ was administered orally. In group 2 , $0.5 \mathrm{mg} / \mathrm{kg}$ of ketamine was administered subcutaneously after the anesthetic induction. In Group 3, or control group, the same amount of normal saline was administered as the placebo, then the surgery got underway.
\end{abstract}

Results: Comparing the administration of oral ketamine, subcutaneous ketamine and normal saline, the study showed that the pain was significantly higher in the placebo group at 30, 60, 90 and 120 minutes. Subcutaneous ketamine resulted in higher delirium at 30,60,90 and 120 minutes than the placebo and oral ketamine. Satisfaction of nurses from the patient's recovery was significantly higher in the oral ketamine group and subcutaneous ketamine group $(\mathrm{p}=0 / 007)$. The need for narcotics in the oral ketamine group and subcutaneous ketamine group was lower than the placebo group $(\mathrm{p}=0 / 001)$. Ketamine administration prolonged the length of stay in the PACU, but it did not increase the frequency of complications such as postoperative nausea and vomiting and laryngospasm or bronchospasm in the two groups during the recovery.

Conclusion: In conclusion, the study indicated that oral administration of low-dose ketamine before surgery can reduce pain severity and spare the need for analgesics after surgery.

Keywords: Ketamine, Delirium, Pain, Pediatric, Surgery

\section{Introduction}

Acute pain is a common complication in children undergoing surgery. Acute pain and delirium are associated with agitated behavior, in which there is a risk that the patient causes harm to himself and others. Acute pain and delirium also dissatisfy parents and caregivers during recovery, prolong the recovery and hospitalization, and make more nursing care necessary. Failure to manage post-operative pain may result in a range of acute and chronic effects. If pain is managed during and after the surgery by intervention in pathophysiological changes during surgeries, fewer complications occur subsequently and the recovery after the surgery and hospital discharge is shortened. Painful stimulus transmission from peripheral nerves to CNS results in endocrine stress response. This leads to sodium and water entrapment and an increase in glucose, free fatty acids, ketone body, and blood lactate. Post-operative pain can suppress the immune system. The associated hyperglycemia can reduce surgical wound healing. Failure to properly handle the pain can activate sympathetic system. The activation leads to a delay in the recovery of gastrointestinal motility, resulting in the occurrence of paralytic ileus. Managing acute post-operative pain and preventing its occurrence can have a significant role in shortening short-term and long-term recovery. Several factors such as age, physical condition, underlying diseases, type of surgery, fear and anxiety and psychological condition play a role in the occurrence and severity of postoperative 
pain; some of them are more pronounced in children, such as age, fear and the anxiety of the separation from parents.

Various medicinal interventions such as the administration of NSAID, acupuncture, ketamine, dexmedetomidine, clonidine, and narcotics effectively prevent the complication, but some issues such as delay in waking up from anesthesia, somnolence and other side effects such as nausea and vomiting can limit the administration of these medications. In another approach, called preemptive therapy, some medicines are administered before the surgery to alleviate pain after surgery. The discovery of NMDA receptor and its role in pain alleviation revolutionized the use of ketamine. The blockage of this receptor can prevent stimulation of nociceptors. As an anesthetic, ketamine is administered for anesthetization, sedation, etc. There are many reports about the effect of low-dose ketamine as an NMDA receptor antagonist on alleviating the pain and reducing the need for systemic narcotics. Ketamine can be administered intravenously, orally, intramuscularly, intranasally, subcutaneously, rectally, and epidurally. Since the medicine is considered a phencyclidine analog, its high doses can have unwanted effects such as hallucination, dysphoria, nightmare, bronchial secretions and intracranial pressure.

Given that the effects of subcutaneous ketamine and oral ketamine on the pain and delirium after surgery has not been studied, this study comparatively investigates the effect of low-dose oral ketamine and subcutaneous ketamine on delirium and post-general anesthesia in children undergoing inguinal hernia.

The occurrence of possible side effects such as nausea and vomiting laryngospasm or bronchospasm in the two groups were compared.

\section{Materials and Methods}

This study is a double-blind, randomized clinical trial to compare two therapies (in three groups). The population were ninety 3-8 year-old patients undergoing inguinal hernia surgery at Children's Hospital and Medical Center during 2014-2015. Each patient should meet the inclusion criteria, including ASA I or II patients, 3-8 years of age, candidate for inguinal hernia and parental consent. Patients having a record of a rare disease, allergies, emergency surgery, mental retardation, genetic diseases and sedative administration were excluded from the study.

The researcher received the consent of parents (Appendix 4) and permission from the Research Deputy of the Tehran Medical University and provided the director of the operating room of Children's Hospital and Medical Center with the documents. To record data, a specially-designed form was used. The data entered into the form is as follows: personally identifiable information, age, gender, weigh, the group name including group 1 (who received $3 \mathrm{cc}$ of $1 \mathrm{mg} / \mathrm{kg}$ ketamine) group 2 (who received $0.5 \mathrm{mg} / \mathrm{kg}$ subcutaneous ketamine) and group 3 (who received normal saline as a subcutaneous placebo), the length of stay in the PACU, the need for analgesics, PAED score and CHEOPS score 30, 60, 90 and 120 minutes after the surgery and SATISFACTION score and ALDRETTE score at the end of the stay in the PACU. Data was registered using direct observation and patients' medical record.

A total of ninety patients aged 3-8 who were candidate for non-emergency inguinal hernia surgery and met inclusion criteria underwent general anesthesia after parental consent was obtained. Monitoring devices and preoxygenation equipment with $100 \% \mathrm{O}_{2}$ were installed at the operating room. Then, a peripheral intravenous line was established. Thereafter, patients were sedated by $2.5 \mathrm{mg} / \mathrm{kg}$ nesdonal, and anesthetic induction was carried out with Sevoflurane $8 \%$. After $1 \mu \mathrm{g} / \mathrm{kg}$ fentalin was received and adequate depth of anesthesia was ensured, tracheal intubation was carried out. Then, anesthesia with 2-3\% Isoflurane and ventilation began. The Patients were randomly divided into three groups based on the random number table. Group 1 received $0.5 \mathrm{mg} / \mathrm{kg}$ subcutaneous ketamine before the surgery. Group 2 received $3 \mathrm{cc}$ of $1 \mathrm{mg} / \mathrm{kg}$ oral ketamine before the surgery. Group 3 received normal saline as a placebo subcutaneously; the administered placebo was at the same volume as subcutaneous ketamine. Thereafter, the surgery got underway and came to the end. When the patients woke up, they were extubated and transferred to the recovery room.

During the recovery and at the post-anesthesia care unit (PACU), a person in charge of recording data entered the following data into a form: pain severity scores, the degree of agitation, patients' readiness for discharge after extubation at 30,60, 90, and 120 minutes, the satisfaction of PACU nurses of the course of recovery, the occurrence of nausea and vomiting and laryngospasm or bronchospasm, and the need for analgesics. If CHEOPS $>10$, the patient would receive $0.5 \mu \mathrm{g} / \mathrm{kg}$ fentalin. Neither patients nor the record keeper was aware of the type of medication. The professor in charge of executing the project was aware of the type of medication.

The data was statistically analyzed by SPSS 22 . The data was represented as the mean, standard deviation, median and range for parametric and non-parametric variables. A comparison was made between the three 
groups in terms of the occurrence of side effects (laryngeal spasms, nausea and vomiting), and the results were analyzed using accurate tests. The t-test was used for parametric data, and the chi-square test was used for non-parametric. P-values less than 0.05 was considered statistically significant.

\section{Results}

A total of 90 patients were divided into three groups; each group consisted of 30 participants. Group 1 received subcutaneous ketamine. Group 2 received oral ketamine. Group 3 received placebo. The mean age was 4.67, 4.40 and 4.67 in the subcutaneous ketamine group, placebo group and oral ketamine group respectively. The p-value was between 0.600 and 0.448 for gender and age respectively. It means that there were no significant differences between the three groups in terms of age and gender (Table 1). As shown in Table 1, the mean weight was 17.47, 16.43 and 16.37 in the subcutaneous ketamine group, placebo group and oral ketamine group respectively. The p-value was 0.452 . It means that there were no significant differences between the three groups in terms weight.

Table 1. Gender, weight and age in control group, subcutaneous ketamine group and oral ketamine group

\begin{tabular}{llll}
\hline Variables & Group & Mean & P-value \\
\hline \multirow{3}{*}{ Gender } & Placebo & 30 patients & 0.600 \\
& Subcutaneous ketamine & 30 patients & \\
age & Oral ketamine & 30 patients & 0.667 \\
& Placebo & 4.40 years old & \\
\multirow{2}{*}{ Weight } & Subcutaneous ketamine & 4.67 years old & 0.452 \\
& Oral ketamine & 4.67 years old & $16.43 \mathrm{~kg}$ \\
& Placebo & $17.47 \mathrm{~kg}$ & $16.37 \mathrm{~kg}$ \\
\hline
\end{tabular}

The length of stay in the PACU was 31.13, 19.43 and 40.67 in the subcutaneous ketamine group, placebo group and oral ketamine group respectively. With regards to the ANOVA test, P-value was 0.000 . It can be concluded that the administration of oral and subcutaneous ketamine prolonged the length of stay in the PACU by $50-100 \%$ (Table 2). In addition, 3 out of 90 patients experienced laryngospasm or bronchospasm; one case was in the subcutaneous ketamine group and the two others were in the oral ketamine group. Two patients in the placebo group, one in the subcutaneous ketamine group and one in the oral ketamine group had nausea and vomiting.

Table 2. Length of stay in PACU in control group, subcutaneous ketamine group and oral ketamine group

\begin{tabular}{lllc}
\hline Variables & Group & Mean & P-value \\
\hline & Placebo & 19.43 days & \\
Length of Stay in PACU & Subcutaneous ketamine & 31.13 days & 0.000 \\
& Oral ketamine & 40.67 days & \\
\hline
\end{tabular}

Table 3. Pain severity based on CHEOPS in control group, subcutaneous ketamine group and oral ketamine group

\begin{tabular}{|c|c|c|c|c|}
\hline Variables & Time & Group & Mean & P-value \\
\hline \multirow{12}{*}{ Pain severity based on CHEOPS } & Cheops 30 & Placebo & 9.37 & \\
\hline & & Subcutaneous ketamine & 8.10 & 0.000 \\
\hline & & Oral ketamine & 7.47 & \\
\hline & Cheops 60 & Placebo & 8.03 & \\
\hline & & Subcutaneous ketamine & 6.67 & 0.000 \\
\hline & & Oral ketamine & 6.60 & \\
\hline & Cheops 90 & Placebo & 7.47 & \\
\hline & & Subcutaneous ketamine & 6.50 & 0.000 \\
\hline & & Oral ketamine & 6.20 & \\
\hline & Cheops 120 & Placebo & 6.77 & 0.000 \\
\hline & & Subcutaneous ketamine & 5.93 & \\
\hline & & Oral ketamine & 5.93 & \\
\hline
\end{tabular}


The pain severity was measured at 30,60,90 and 120 minutes based on CHEOPS; its results are shown in Table 3 . The results indicated a significant difference between the pain severity in the three groups at all times. Oral ketamine had the most positive effects on pain relief after the surgery compared with placebo and subcutaneous ketamine.

The delirium severity was measured at 30, 60, 90 and minutes based on PAED. The P-values were less than 0.05 at all times. It indicates that there were significant differences between the three groups in terms of delirium severity based on PAED (Table 4).

Table 4. Delirium severity based on PAED in control group, subcutaneous ketamine group and oral ketamine group

\begin{tabular}{|c|c|c|c|c|}
\hline Variables & Time & Group & Mean & P-value \\
\hline \multirow{12}{*}{ Delirium severity based on PAED } & \multirow[t]{3}{*}{ PAED 30} & Placebo & 10.87 & \\
\hline & & Subcutaneous ketamine & 12.43 & 0.000 \\
\hline & & Oral ketamine & 11 & \\
\hline & \multirow[t]{3}{*}{ PAED 60} & Placebo & 5.57 & \\
\hline & & Subcutaneous ketamine & 5.27 & $000 / 0$ \\
\hline & & Oral ketamine & 4.60 & \\
\hline & \multirow[t]{3}{*}{ PAED 90} & Placebo & 2.13 & \\
\hline & & Subcutaneous ketamine & 3.73 & 0.000 \\
\hline & & Oral ketamine & 2.73 & \\
\hline & \multirow[t]{3}{*}{ PAED 120} & Placebo & 1.33 & 0.000 \\
\hline & & Subcutaneous ketamine & 0.73 & \\
\hline & & Oral ketamine & 0.53 & \\
\hline
\end{tabular}

At the time of discharge, the degree of nurse's satisfaction of the recovery was determined by the RN Satisfaction Score. The mean was 3.30, 3.83 and 3.70 for the placebo group, subcutaneous ketamine group and oral ketamine group respectively (Table 5). As seen in Table 5, the P-value was $0.007(<0.05)$. It means that the difference between the three groups in terms of nurse's satisfaction of the recovery was significant. Subcutaneous ketamine resulted in more satisfaction than placebo and oral ketamine.

Table 5. PACU nurse's Satisfaction Score of the recovery in control group, subcutaneous ketamine group and oral ketamine group

\begin{tabular}{llll}
\hline Variables & Group & Mean & P-value \\
\hline & Placebo & 3.30 \\
PACU nurse's Satisfaction Score of the recovery & Subcutaneous ketamine & 3.83 & 0.007 \\
& Oral ketamine & 3.70 & \\
\hline
\end{tabular}

The patients of each group whose pain score was above 10 based on the CHEOPS received fentanil as an analgesic. In the placebo group, 12 patients received fentanil, since their score was above 10 . In the subcutaneous ketamine group the number of patients who received it was 3. In the oral ketamine group, just one patient needed it.

\section{Discussion}

This comparative-descriptive study investigated 90 pediatric patients, who were divided into three groups; one group received ketamine subcutaneously, one group received it orally and one group received placebo. Each group consisted of 30 patients. The purpose of the study was to investigate the effects of subcutaneous ketamine and oral ketamine on the postoperative pain and agitation of pediatric patients undergoing inguinal hernia. To that end, $0.5 \mathrm{mg} / \mathrm{kg}$ subcutaneous ketamine and $1 \mathrm{mg} / \mathrm{kg}$ oral ketamine was administered before surgery.

As seen in the result section, no significant difference was observed between the tree groups in terms of age, gender and weight. It seems that the tree groups were homogeneous, and there had been no disruptive factors in the study in terms of demographic variables.

Expected complications such as the occurrence of nausea and vomiting and laryngospasm or bronchospasm were not different in the three groups. The use of ketamine, either subcutaneously or orally, and placebo did not affect the occurrence of these complications. 
The length of stay in the recovery and the condition of patients during the recovery were two other issues investigated in the study; the latter was measured to check whether the patients could meet the discharge criteria. It was observed that oral ketamine prolonged the former, but oral ketamine resulted in the better course of recovery. In fact, oral administration of ketamine led to longer stay in recovery.

In terms of severity of pain, which was the factor of interest in this study, the oral ketamine group experienced the least pain at 30,60, 90 and 120 minutes based on the CHEOPS. The need for sedatives was significantly lower in the oral ketamine group than in the placebo group and subcutaneous ketamine group. In the subcutaneous ketamine group, the nurse's satisfaction of the course of recovery was higher than the other groups.

On the other hand, delirium, which is associated with the administration of anesthesia, was higher in the subcutaneous ketamine group than oral ketamine and placebo. It was seemingly independent of the delirium resulting from post-operative pain. Probably, it was associated with the way ketamine was administered. There was no significant relationship between pain and delirium at 30,60, 90 and 120 minutes between the three groups. This suggests that the delirium experienced by the patients did not result from post-operative pain.

The body of literature investigating the issue has found that ketamine is effective in the reduction of post-operative pain. However, many studies indicate that administration of ketamine before surgery has no effect on the post-operative pain.

The amount of ketamine and the way it has been administrated might have been different in these studies. For example, Dix et al. (2013) studied the administration of ketamine, but the injection was performed intravenously. Javid et al. (2012) studied the post-surgical injection of the same dose of subcutaneous ketamine as administered in our study. Nakao et al. (1999) found that intravenous injection of ketamine before surgery might alleviate pain and that post-surgical administration of ketamine does not have such effect. As mentioned above, the effects of ketamine depends largely on the amount, time and method of administration.

Bell (2006) studied the complications experienced after the administration of ketamine. They found that ketamine reduces nausea and vomiting. The difference between the three groups was not significant in terms of the occurrence of nausea and vomiting. Safavi et al. (2011) and Javid et al. (2012) both obtained the same result in terms of post-surgical effects of ketamine on alleviating the pain experienced by children. In the former study, the researchers employed visual analog scale to measure the level of pain; in the latter study, the researchers employed CHEOPS. None of them investigated delirium or other factors assessed in our study.

Dix et al. (2003) found that ketamine administration does not alleviate pain after surgery and that it increases hallucination, but they administered high doses of ketamine compared with our study. Bazin et al. (2010) found that ketamine does not have any effect on post-surgical pain, but the dose administered in their study was $0.15 \mathrm{mg} / \mathrm{kg}$ and the age of children ranged from 6 months to 5 years.

\section{Conclusion}

In conclusion, the study indicated that oral administration of low-dose ketamine before pediatric surgery can alleviate pain severity, reduce delirium and spare the need for analgesics during recovery, and that the subcutaneous administration of low-dose ketamine can increase the nurse's satisfaction of the course of recovery. Ketamine did not increase the occurrence of unwanted complications such as nausea and vomiting and laryngospasm or bronchospasm.

\section{Acknowledgment}

This project was implemented in cooperation with Tehran Pediatric Medical Center Hospital. We should express our appreciation towards the esteemed head of the hospital for allowing the access to the pediatric patients. We should express our appreciation toward the Research Deputy of Tehran University of Medical Sciences for financially supporting the project.

\section{Conflict of interests}

The authors declare that there is no conflict of interests regarding the publication of this paper.

\section{References}

Bazin, V., Bollot, J., Asehnoune, K., Roquilly, A., Guillaud, C., De Windt, A., ... \& Lejus, C. (2010). Effects of perioperative intravenous low dose of ketamine on postoperative analgesia in children. European Journal of Anaesthesiology (EJA), 27(1), 47-52.

Becke et al. (2005). Intraoperative low-dose S-ketamine has no preventive effects on postoperative pain and morphine consumption after major urological surgery in children. Pediatric Anesthesia, 15(6), 484-90. 
Behdad, A., Hosseinpour, M., \& Khorasani, P. (2011). Preemptive use of ketamine on post operative pain of appendectomy. The Korean Journal of Pain, 24(3), 137-40.

Bell, R. F., Dahl, J. B., Moore, R. A., \& Kalso, E. (2006). Perioperative ketamine for acute postoperative pain. Cochrane Database Syst Rev., 1(1).

Dahmani et al. (2011). Ketamine for perioperative pain management in children: A meta-analysis of published studies. Pediatric Anesthesia, 21(6), 636-52.

Dix, P., Martindale, S., \& Stoddart, P. (2003). Double-blind randomized placebo-controlled trial of the effect of ketamine on postoperative morphine consumption in children following appendicectomy. Pediatric Anesthesia, 13(5), 422-6.

Eide, P. K., Stubhaug, A., \& Øye, I. (1995). 8 The NMDA-antagonist ketamine for prevention and treatment of acute and chronic post-operative pain. Baillière's Clinical Anaesthesiology, 9(3), 539-54.

Elia, N., \& Tramer, M. R. (2005). Ketamine and postoperative pain-a quantitative systematic review of randomised trials. Pain, 113(1-2), 61-70.

Hajipour, A. (2002). Effects of preemptive Ketamine on post-cesarean analgesic requirement. Acta Medica Iranica, 40(2), 100-3.

Hazama, K., Nakao, M., Kawaguchi, R., Nakatani, K., Nakagawa, M., \& Unetani, H. (1999). Pre-incisional administration of ketamine reduced the postoperative pain. Masui The Japanese Journal of Anesthesiology, 48(12), 1302-7.

Himmelseher, S., \& Durieux, M. E. (2005). Ketamine for perioperative pain management. Anesthesiology: The Journal of the American Society of Anesthesiologists, 102(1), 211-20.

Javery, K. B., Ussery, T. W., Steger, H. G., \& Colclough, G. W. (1996). Comparison of morphine and morphine with ketamine for postoperative analgesia. Canadian Journal of Anaesthesia, 43(3), 212-5.

Javid, M. J., Hajijafari, M., Hajipour, A., Makarem, J., \& Khazaeipour, Z. (2012). Evaluation of a low dose ketamine in post tonsillectomy pain relief: A randomized trial comparing intravenous and subcutaneous ketamine in pediatrics. Anesthesiology and Pain Medicine, 2(2), 85.

Pieters, B. J., Penn, E., Nicklaus, P., Bruegger, D., Mehta, B., \& Weatherly, R. (2010). Emergence delirium and postoperative pain in children undergoing adenotonsillectomy: A comparison of propofol vs sevoflurane anesthesia. Pediatric Anesthesia, 20(10), 944-50.

Safavi, M., Honarmand, A., \& Nematollahy, Z. (2011). Pre-incisional analgesia with intravenous or subcutaneous infiltration of ketamine reduces postoperative pain in patients after open cholecystectomy: A randomized, double-blind, placebo-controlled study. Pain Medicine, 12(9), 1418-26.

Subramaniam, K., Subramaniam, B., \& Steinbrook, R. A. (2004). Ketamine as adjuvant analgesic to opioids: A quantitative and qualitative systematic review. Anesthesia \& Analgesia, 99(2), 482-95.

Tan, P. H., Cheng, J. T., Kuo, C. H., Tseng, F. J., Chung, H. C., Wu, J. I., ... \& Yang, L. C. (2007). Preincisional subcutaneous infiltration of ketamine suppresses postoperative pain after circumcision surgery. The Clinical Journal of Pain, 23(3), 214-218.

\section{Copyrights}

Copyright for this article is retained by the author(s), with first publication rights granted to the journal.

This is an open-access article distributed under the terms and conditions of the Creative Commons Attribution license (http://creativecommons.org/licenses/by/4.0/). 